\title{
Usefulness of the ManageMed Screen (MMS) and the Screening for Self-Medication Safety Post Stroke (S5) for Assessing Medication Management Capacity for Clients Post-Stroke
}

Jessica J. Bolduc

Mercy Hospital - Portland, jbolducot@gmail.com

Regula H. Robnett

University of New England

Follow this and additional works at: https://nsuworks.nova.edu/ijahsp

Part of the Occupational Therapy Commons

\section{Recommended Citation}

Bolduc JJ, Robnett RH. Usefulness of the ManageMed Screen (MMS) and the Screening for SelfMedication Safety Post Stroke (S5) for Assessing Medication Management Capacity for Clients PostStroke. The Internet Journal of Allied Health Sciences and Practice. 2015 Apr 01;13(2), Article 3.

This Manuscript is brought to you for free and open access by the College of Health Care Sciences at NSUWorks. It has been accepted for inclusion in Internet Journal of Allied Health Sciences and Practice by an authorized editor of NSUWorks. For more information, please contact nsuworks@nova.edu. 


\title{
Usefulness of the ManageMed Screen (MMS) and the Screening for Self- Medication Safety Post Stroke (S5) for Assessing Medication Management Capacity for Clients Post-Stroke
}

\begin{abstract}
Occupational therapists need to efficiently and accurately screen a client's medication management capacity, especially for clients post-stroke. Most therapists are not aware of, nor do they utilize specific assessments for, medication management capacity. The purpose of this pilot study was to compare the results of the ManageMed Screen (MMS), the Screening for Self-Medication Safety Post Stroke (S5), and the Montreal Assessment of Cognition (MoCA) on a population of rehabilitation clients post-stroke to determine the usefulness of the medication assessment tools in clinical practice. These screens were designed for use in occupational therapy practice among other healthcare professions: the MMS was validated for the general adult population, the S5 for clients post-stroke, and the MoCA is a cognitive screen used with adult clients with a variety of diagnoses including stroke. The MoCA was used to explore the potential relationship between cognition and medication management capacity. Study participants included five clients post-stroke and three occupational therapists. Clients were screened by the occupational therapists with the MMS, S5, and MoCA, and clinicians also participated in a focus group to assess their perceived usefulness of the screens. Results demonstrated that the MMS score compared to the $\mathrm{S} 5$ score was not statistically significant $(r=.671, p=.215)$. There is no established consistency between the MoCA and MMS given these five clients. The MMS score was correlated to the MoCA score and was not found to be significant at a value of .205 with $p=.741$. The S5 score was also correlated to the MoCA score using SPSS and was found to have a non-significant value of -.287 and $p=.640$. Additionally, through a focus group, clinicians deemed both the MMS and S5 as useful, but felt the MMS was a more useful screen for their clinical practice with regard to efficient and practical use with clients post-stroke in a rehabilitation setting.
\end{abstract}




\title{
IJAHSP \\ The Internet Journal of Allied Health Sciences and Practice
}

Dedicated to allied health professional practice and education

Vol. 13 No. 2 ISSN 1540-580X

\section{Usefulness of the ManageMed Screen (MMS) and the Screening for Self- Medication Safety Post Stroke (S5) for Assessing Medication Management Capacity for Clients Post-Stroke}

\author{
Jessica J. Bolduc, DrOT, MSOTR/L1 \\ Regula H. Robnett, PhD, OTR/L2
}

1. Staff Occupational Therapist, Mercy Hospital, Portland, Maine

2. Professor, Occupational Therapy Department, University of New England, Portland, Maine

United States

\begin{abstract}
Occupational therapists need to efficiently and accurately screen a client's medication management capacity, especially for clients post-stroke. Most therapists are not aware of, nor do they utilize specific assessments for, medication management capacity. The purpose of this pilot study was to compare the results of the ManageMed Screen (MMS), the Screening for SelfMedication Safety Post Stroke (S5), and the Montreal Assessment of Cognition (MoCA) on a population of rehabilitation clients post-stroke to determine the usefulness of the medication assessment tools in clinical practice. These screens were designed for use in occupational therapy practice among other healthcare professions: the MMS was validated for the general adult population, the S5 for clients post-stroke, and the MoCA is a cognitive screen used with adult clients with a variety of diagnoses including stroke. The MoCA was used to explore the potential relationship between cognition and medication management capacity. Study participants included five clients post-stroke and three occupational therapists. Clients were screened by the occupational therapists with the MMS, S5, and MoCA, and clinicians also participated in a focus group to assess their perceived usefulness of the screens. Results demonstrated that the MMS score compared to the S5 score was not statistically significant $(r=.671, p=.215)$. There is no established consistency between the MoCA and MMS given these five clients. The MMS score was correlated to the MoCA score and was not found to be significant at a value of .205 with $p=.741$. The S5 score was also correlated to the MoCA score using SPSS and was found to have a non-significant value of -.287 and $p=.640$. Additionally, through a focus group, clinicians deemed both the MMS and S5 as useful, but felt the MMS was a more useful screen for their clinical practice with regard to efficient and practical use with clients post-stroke in a rehabilitation setting.
\end{abstract}

\section{INTRODUCTION}

The capacity for medication management is of eminent concern for health professionals as there is an increase in chronic disease and medication dependency among older adults. ${ }^{10,12}$ Poor medication management is related to an increased number of medication errors, hospital admissions, and higher mortality rates in the older adult and those with chronic health issues, such as stroke.6,7 Medication management capacity is the result of intact higher level cognitive skills which enable a person to be independent and safe with this set of activities of daily living (ADL) and instrumental activities of daily living (IADL) tasks. Occupational therapists are charged with assessing medication management capacity as it can impact independence and safety with daily functioning.

Best practice in occupational therapy would indicate the use of comprehensive and psychometrically strong tools for client assessment when available. ${ }^{1}$ In current practice, specifically in the rehabilitation setting, one often will find clinicians using clinical inference from observation of ADLs or "home grown" screens or questionnaires to assess medication management capacity rather than standardized assessments. ${ }^{11}$ Health professionals, such as occupational therapists, are not necessarily accurate at predicting a client's ability with medication management based on observation; functional ability of this task is not always detected in routine assessments or observations. ${ }^{6}$ Wales et al found that occupational therapists often do not use standardized 
assessment in practice due to their lack of readiness or skill, time commitments, "motivation, self-confidence, lack of support from management, personal values and beliefs and lack of knowledge" or awareness of the availability of assessments (p. 2). ${ }^{16}$ Clinical judgment about performance of functional tasks, such as medication management, when completed in an unstructured and non-standardized manner, leads to decreased legitimacy and limited contributions to evidence-based outcomes in occupational therapy.5,6 Using standardized and normed instruments in clinical practice can assist with efficiency and documentation of changes in status, and improves overall effectiveness of occupational therapy practice. ${ }^{4}$ However, the approach to medication management capacity screening traditionally has not been standardized in occupational therapy, most likely due to lack of awareness of available screens and/or non-availability of screens for a specific population, such as clients post-stroke.

There are about a dozen screens in the open market labeled as medication management capacity screens; however, upon further review of these screens, many are not appropriate for use with clients post-stroke as they lack research on this population, and components within the screen that assess cognitive and physical skills of clients post-stroke are lacking (Appendix A). The literature also indicates that assessing cognition alone does not equate to an accurate screen for medication management capacity, and for clients post-stroke, physical performance must also be assessed. ${ }^{3}$ Some of the screens only address cognition. Furthermore, many of these screens are profession specific (not occupational therapy), diagnosis specific (not stroke), or do not measure medication capacity, but rather adherence patterns.

It would be beneficial for occupational therapists to have a means to efficiently and accurately screen a client's medication management capacity, especially for clients post-stroke. Most therapists are not aware of, nor do they utilize, specific assessments for medication management capacity, partly due to lack of readily available fitting assessments. The purpose of this study was to determine the capacity to follow a medication routine based on a client's cognitive and physical performance (not their choice to adhere to their own prescribed medication routine). For a client post-stroke, the ability to manage medications can be impacted by changes in communication skills, physical abilities, cognition, behavior, sensation, and visual-perception. ${ }^{8}$ Up to $65 \%$ of stroke survivors demonstrate changes in cognitive function including attention deficits, memory deficits, and spatial neglect, all of which can impact functional recovery and safety with medication management.3,17 To ensure clients post-stroke have the physical and cognitive skills for safe medication management, screens that assess these skills and are valid and reliable for this population are needed.

Two medication management screens have been developed and were found to be appropriate for use in occupational therapy practice: the ManageMed Screen (MMS) and the Screening for Self-Medication Safety Post Stroke (S5).8,12 The MMS and S5 were developed and meet the need for occupational therapists by addressing cognitive and physical performance skills required for medication management. The MMS was specifically developed by occupational therapists for assessing medication management in the general adult population by health professionals; it was found to be able to differentiate between adults who need assistance and those who are independent with this task..$^{12}$ The MMS is standardized and has undergone validation studies as well. ${ }^{12}$ The S5 was developed by occupational therapists for health professionals to assess medication management capacity for clients post-stroke. ${ }^{8}$ The S5 has undergone one pilot test by its authors for use with adult clients post-stroke; thus, the 55 has limited data to support its reliability and validity. These screens continue to need clinical research to support their use in specific client populations such as stroke. In a rehabilitation setting for clients post-stroke, clinicians need at least one practical screen that will accurately and efficiently assess each client's cognitive and physical skills required to manage their medications.

The Montreal Cognitive Assessment (MoCA) assesses the clients' cognition and explores the relationship of cognition and medication management capacity. The MoCA is a performance-based screen to assess cognitive skills which contains 16 test items that examine attention and concentration, executive functions, memory, language, visuoconstructional skills, conceptual thinking, calculations, and orientation. ${ }^{9}$ The MoCA has undergone several years of research and study findings have consistently demonstrated excellent validity and reliability. ${ }^{15}$

\section{RESEARCH QUESTIONS}

1. Is there a correlation between the ManageMed Screen (MMS) and the Screening for Self-Medication Safety Post-Stroke Scale (S5) scores for assessing capacity in medication management?

2. Is there a correlation between the ManageMed Screen (MMS) and the Screening for Self-Medication Safety Post Stroke Scale (S5) scores compared to the Montreal Cognitive Assessment (MoCA) for assessing cognition as an indicator for capacity in medication management?

3. Which of the medication management screening tools (or aspects of each screen) offer the most clinically relevant information to help inform decision making for treatment and discharge planning for medication management capacity for occupational therapy practitioners?

(C) The Internet Journal of Allied Health Sciences and Practice, 2015 


\section{METHODOLOGY}

This pilot study used quantitative and qualitative methodologies to begin to determine the usefulness of two fairly new medication management screens for use in occupational therapy practice. The scores of the ManageMed Screen (MMS), the Screening for Self-Medication Safety Post Stroke (S5), and the Montreal Assessment of Cognition (MoCA) were compared on a population of rehabilitation clients post-stroke to determine their usefulness in clinical practice. Part I of the study included five clients poststroke and three occupational therapists. Clients were screened by the occupational therapists with the MMS, the S5, and the MoCA. Part II consisted of the clinicians who participated in a focus group to assess their perceived usefulness of the screens from part I.

\section{PARTICIPANTS}

Clinician and client participants were recruited from New England Rehabilitation Hospital Portland, an inpatient rehabilitation hospital in Maine. Institutional Review Board approval for this project was obtained from Nova Southeastern University. Clinicians included one male and two females with clinical experience ranging from 3 to 15 years. Five clients participated in the study, including three females and two males with a mean age of 72.6 years. The post stroke clients were 72 hours from discharge, had a diagnosis of stroke, scored a 4 or greater on the Functional Independence Measure for cognition, and were able to speak and read English. Twenty percent of client participants had a right hemispheric stroke of embolic origin, days poststroke ranged from 9 to 17 with a mean of 11.6 days, and clients were taking medications ranging from 4 to 13 medications with an average of 9.8 medications at the time of screen administration.

\section{INSTRUMENTATION}

The ManageMed Screen (MMS) has 30 questions and performance tasks to assess "reading, medication knowledge base, problem solving, short-term and prospective memory, and calculations" (p. 2). ${ }^{13}$ The MMS is standardized and has evidence of adequate validity and reliability. A sample question for reading, medication knowledge base, and problem solving is "If this [prescription vial A] were a prescription from your doctor, would it be safe to take?" The client is given a prescription bottle with printed label information to review; the client is to note the date the medication expired. Other questions related to problemsolving, short-term memory, calculation, and physical skills include "Can you open this container? Open it and count the number of pills. How long will these pills last if taken as prescribed?" Lastly, one IADL task in particular requires the client to successfully utilize higher level cognitive skills and assesses physical skills when the client is asked to set up a pill organizer using the three medications based on instructions on each prescription bottle while recalling that their meals are taken at 8:00am, noon and 5:30pm. Slots in a plastic organizer must be opened and the client is asked to put in the correct number of pills at the correct times. Performance on the MMS is scored on a 0 or 1 scale for each item, with 0 indicating unable to perform or performed incorrectly and 1 as performed correctly. The maximum score one can obtain is 39 points; the client's score can then be compared to age-related norms developed by the authors through previous research.

Potential impairments that can be distinguished with the use of this screen include decreased vision, decreased prospective memory, decreased recognition, decreased safety awareness, decreased attention to detail, decreased physical ability, decreased calculation skills, decreased retrospective memory, decreased organizational skills, and decreased insight. ${ }^{13}$ The findings of these impairments could indicate poor medication management capacity and also provide areas for the occupational therapist to focus on either through rehabilitation or remediation intervention strategies.

The Screening for Self-Medication Safety Post Stroke (S5) has a checklist of 16 questions to assess basic orientation, the manipulation of medication bottles, calculating medication doses, immediate and delayed memory recall tasks, manipulating a syringe (if appropriate for the client), completing visual recognition and visual spatial tasks, and problem-solving questions to assess cognition (orientation; immediate and delayed memory recall), communication (comprehension; reading), motor function, visual-perception, and judgment/executive functions/self-efficacy.8,14 A sample question which addresses calculation, visual recognition, and problem solving includes, "If you have to take 2 pills in the morning and 2 at night, show me how you would group the pills." This is stated after providing the client an open bottle with 8 identical white disc-shaped pills. To assess physical skills, some test items include providing a syringe without a needle and asking the client to demonstrate how to inject their medications or providing the client with a bottle of liquid medication and asking them to "Open the bottle and pour out $10 \mathrm{ml}$ of the liquid into this cup." Scoring is completed by indicating if the task was done correctly by checking the Yes or No boxes beside each question. The score is out of 16 questions; in general, a higher score of more "Yes" responses would indicate less difficulty with medication management. More importantly, the screen offers specific areas of concern (when a question is marked as "No") that may be able to be addressed in occupational therapy intervention. Scoring criteria was not developed beyond a yes/no checklist. For the purpose of this study as developed by the primary investigator, questions marked "yes" were counted as 1 point. If a client scored a 14,15 or 16 on the $\mathbf{S}$, this was deemed "normal" performance capacity. If a client scored 10 to 13 , this

(C) The Internet Journal of Allied Health Sciences and Practice, 2015 
was equated to "questionable" performance capacity, and if a client scored 9 or less, this was equated to a "poor" performance capacity with medication management.

The Montreal Cognitive Assessment (MoCA) contains 16 test items that examine attention and concentration, executive functions, memory, language, visuoconstructional skills, conceptual thinking, calculations, and orientation. ${ }^{9}$ A sample question from version 2 to assess attention, concentration, executive function, visuoconstructional skills, and conceptual thinking is drawing a clock. The client is asked to draw a clock, place all numbers in the clock and set the time to five past four. ${ }^{9}$ The client is scored based on contour of the clock, correct sequence, and placement of numbers in the clock along with correct placement of the hands of the clock. Points are given based on correct answers/performance of task. Clinician participants were trained by the primary investigator for all three assessments, and inter-rater reliability was established by comparing scores on a simulated client.

In part II of the study, the instrument used in the guided focus group for clinician participants was an open-ended interview guide of 10 questions devised by the primary investigator. The questions used engagement and exploration questions to elicit feedback on administration of the screens as suggested by Creswell. ${ }^{2}$ The semi-structured questions were intended to evoke dialogue among the clinicians and to solicit feedback regarding their impression of the screens, the value of information obtained from testing, advantages or disadvantages of each screen, scoring discrepancies or questions, and overall usefulness the screens. A sample question included, "Share your impression or feedback on the administration of each test, such as ease of setup, clarity of instructions, items in test kits, questions asked etc."

\section{Data Collection Part I}

Data collection lasted 6 weeks for a minimum of a sample of convenience of five client participants. Clients were selected following the inclusion criteria and consented to participate. Clinicians gathered data in order of the questions as listed on the data collection sheets. Information such as age, gender, hand dominance, stroke location/type, days post-stroke, medication questions, presence of visual impairment, and ratings for stress and sleep were always completed first and in numerical order. The screens were given in random order for each client.

\section{Data Collection Part II}

After the clinicians completed a minimum of five assessments and after six weeks from the start of data collection, the clinicians participated in a 1 hour and 30 minute focus group interview. The interview was transcribed verbatim by hand by the primary investigator during the interview process. The primary investigator facilitated the discussion with the focus on clinician feedback on administration of each screen (ease, setup, instructions etc.), value of data obtained from the screens, advantage/ disadvantage of each screen, and usefulness of the screens to clinical practice.

\section{Data Part I}

As seen in Table 1, compared to the normative scores by age, client 1 scored within one standard deviation above the mean score for the MMS, "normal" performance on the S5, and below the mean score for the MoCA. Client 2 scored well above one standard deviation of the mean score for the MMS, "normal" performance on the S5, and above the mean score for the MoCA. Client 3 scored within one standard deviation below the mean score on MMS, "questionable" performance on the S5, and at the mean for the MoCA. Client 4 scored within one standard deviation below the mean score for the MMS, "normal" performance on the S5, and below the mean for the MoCA. Client 5 scored within one standard deviation above the mean score for the MMS, "normal" performance on the S5, and at the norm for the MoCA. 
Table 1. Client Scores on MMS, S5, and MoCA

\begin{tabular}{|c|c|c|c|}
\hline Client & $\begin{array}{c}\text { MMS } \\
\text { (mean mean +/- SD* }\end{array}$ & $\mathbf{S 5}^{* *}$ & MoCA $^{* * *}$ \\
\hline 1 & $\begin{array}{c}30 \\
(29+/-3.39)\end{array}$ & 15 & 21 \\
\hline 2 & $\begin{array}{c}35 \\
(29+/-3.39)\end{array}$ & 15 & 28 \\
\hline 3 & 26 & 13 & 26 \\
\hline 4 & $(29+/-3.39)$ & 15 & 26 \\
\hline 5 & $\begin{array}{c}32 \\
(34+/-3.29)\end{array}$ & 14 & \\
\hline
\end{tabular}

${ }^{*}$ MMS age norms based on client age $+/$ - standard deviation (SD) ${ }^{13}$

${ }^{* *}$ S5:14, 15 or 16 = 'normal' performance capacity;

10 to $13=$ 'questionable' performance capacity;

9 or less $=$ 'poor' performance capacity ${ }^{8}$

${ }^{* * *}$ MoCA: 26 or better $=$ normal performance ${ }^{9}$

\section{RESULTS PART 1}

Using visual comparison of data and statistical data analysis presented in the above sections, the question, "How consistent are the ManageMed Screen (MMS) and the Screening for Self-Medication Safety Post Stroke Scale (S5) scores for assessing capacity in medication management?" can be answered by reviewing data in Table 2. Overall, all clients scored within their age norms on the MMS, and all but client 3 scored "normal" performance capacity on the S5. Using the Statistical Package for Social Sciences (SPSS - version 20), each test score was correlated using nonparametric two-tailed test of Spearman's rho correlations. The MMS score was compared to the $S 5$ score and was found to be statistically non-significant $(r=.671, p=.215)$.

Table 2. Score Comparison of the MMS and S5

\begin{tabular}{|l|c|c|}
\hline Client & $\begin{array}{c}\text { MMS Score } \\
\text { (mean score +/- SD)* }\end{array}$ & S5 Score $^{* *}$ \\
\hline 1 & 30 & 15 \\
\hline 2 & $(29+/-3.39)$ & 15 \\
\hline 3 & 35 & 13 \\
\hline 4 & $(29+/-3.39)$ & 15 \\
\hline 5 & 26 & 14 \\
\hline
\end{tabular}

${ }^{*}$ MMS age norms based on client age $+/$ - standard deviation (SD) ${ }^{13}$

${ }^{* *} \mathrm{~S} 5: 14,15$ or 16 = 'normal' performance capacity;

10 to 13 = 'questionable' performance capacity;

9 or less $=$ 'poor' performance capacity ${ }^{8}$

When comparing the client's performance on the MMS to the S5, the clients who scored the highest on the MMS also scored highest on the S5 (e.g. Clients 2 and 4 in Table 2). However, this pattern is not consistent as clients 1, 3, and 5 also performed within the norm on the MMS and clients 1 and 5 scored 14 or 15 on the S5 suggesting "normal" performance capacity. Nonetheless, client 3 who scored the lowest on the MMS (but still within age norms) also scored the lowest on the S5 ("questionable" performance capacity). This pattern does not suggest a true pattern of consistency between the MMS and S5 scores. 
To answer the second research question, "Is there a correlation between the ManageMed Screen (MMS) and the Screening for Self-Medication Safety Post Stroke Scale (S5) scores compared to the Montreal Cognitive Assessment (MoCA) for assessing cognition as an indicator for capacity in medication management?" the data are presented in Table 1.

When comparing client scores from the MMS and the MoCA, there is no established consistency between the MoCA and MMS given these five clients.

When comparing the MoCA to the S5, clients 1 and 5 who scored below norms on the MoCA scored within "normal" performance on the S5; conversely, client 3 who scored "questionable" performance on the S5 scored at the norm on the MoCA. There does not appear to be a consistent relationship between the S5 and the MoCA.

Using Statistical Package for Social Sciences (SPSS - version 20), the test scores for the MMS and MoCA were correlated using nonparametric two-tailed test of Spearman's rho correlations. The MMS score was correlated to the MoCA score and was not found to be significant at a value of 205 with $p=.741$. The S5 score was also correlated to the MoCA score using SPSS and was found to have a non-significant value of -.287 and $p=.640$.

From the comparison of the both the MMS and S5 to the MoCA for consistency of scoring, there is not a consistent relationship with scores for medication management capacity and cognition. Normal performance based on norms of the MMS does not necessarily indicate "normal" performance on the MoCA, much like "normal" performance on the S5 does not indicate "normal" performance on the MoCA. Any relationships noted must be interpreted with the utmost caution due to the pilot nature of this study.

\section{DATA PART II}

Thematic qualitative analysis from the transcribed focused group data was completed for data analysis of part II. Thematic analysis of all 10 questions and answers led to the development of general themes that enables this data presentation. Theme $\mathrm{A}$ entails clinician feedback on usefulness of the screens via administration, ease of setup, clarity of instructions, test kit assembly/use, and advantages/disadvantages of each screen. Theme $B$ relates the use of the screens in clinical practice via the value of the data collected for treatment planning and discharge recommendations, and clinicians' impression with the screen's ability to differentiate adequate from inadequate medication management skills. Theme $\mathrm{C}$ relates to the clinicians preference of screen for future use and the subjects' responses to testing.

\section{RESULTS PART II}

The three clinicians' consensus revealed that they felt the MMS was the more useful screen for medication management when comparing advantages and disadvantages in regards to accuracy, efficiency, practicality, and clinical relevance. This directly answers the third research question, "Which of the medication management screening tools (or aspects of each screen) offers the most clinically relevant information to help inform decision making for treatment and discharge planning for medication management capacity for occupational therapy practitioners?" While the MMS took the longest to administer, previously mentioned advantages appear to make it the most clinically useful tool for clients post-stroke in this setting when compared to the S5 and MoCA. The MMS offers more clinically relevant information by screening visual, cognitive, and physical performance skills by having clients read medication information and pill bottles, manipulate pill bottles and pills, and complete memory and problem solving tasks. The IADL component of filling of the pill organizer of the MMS was the most revealing in terms of where clients could have difficulty with managing a complex medication routine. However, it was also this component that was the most challenging for clinicians to administer and score.

Clinicians also felt that they would adopt the use of the MoCA in their clinical practice, but not as a medication management capacity screen. The MoCA provides clinically relevant information as it can detect what areas of cognition are challenges for the client (visuospatial/executive, naming, memory, attention, language, abstraction, and/or orientation). The use of the MoCA can target treatment towards compensation or remediation approaches addressing the identified deficits. Additionally, the MoCA is well understood by the other interprofessional team members and can create a dialogue during team rounds to ensure the client is receiving the necessary services while in rehabilitation.

The S5 was the least likely of the screens to be frequently used by these clinicians as it currently lacks usefulness and clinically relevant information. The S5 was found to be too subjective, vague and the overall score does not relate to a norm. Generally, clinicians felt that while the S5 was the quickest and simplest screen to administer, it did not yield data with enough depth to be useful. Clinicians did appreciate that a variety of pill sizes, colors, and pill or liquid bottles were included in the screen, but the score was not viewed as meaningful. The S5 will continue to be a possible tool that clinicians can offer a client should the MMS

(C) The Internet Journal of Allied Health Sciences and Practice, 2015 
be too challenging or if the client is resistive to the screening process. This qualitative, thematic analysis based on clinician feedback suggests that overall, the MMS offers the most clinically useful and relevant information for treatment and discharge planning.

\section{DISCUSSION}

Best practice would indicate the use of comprehensive and psychometrically strong tools for client assessment; clinicians often choose assessments tools based on their usefulness and practicality to their practice. ${ }^{1}$ From the literature, clinicians were made aware of several medication management screens available on the market for use in practice. However, clinicians were also informed of key items that should be included in a medication management screen, such as language abilities, reading and writing skills, numeric/calculation capabilities, limb praxis, visuospatial function, social use of language, emotional function, attention, executive function, memory, and observation of performance of medication management tasks from the literature of Donovan et al and Robnett et al.,12 When comparing these key items to current assessments, the only medication management capacity screens remaining that fit these criteria were the MMS and S5. The MMS and S5 were chosen for further study because of their content and format that included cognitive and physical assessments.

The results from this pilot study have introduced data for the ManageMed Screen and the Screening for Self-Medication Safety Post Stroke for assessing medication management capacity for clients post-stroke. While statistical significance of scores between the MMS and S5 was not obtained, cognition is one construct in medication management capacity that can be assessed with the MoCA which enables the clinician to determine what area of cognition may be impacting performance with medication management.

The second focus of the study utilized qualitative analysis of clinician responses for the usefulness of the medication management screens in clinical practice. General consensus is that occupational therapy practitioners preferred the use of MMS due to the depth and breadth of information the screen was able to provide. This information aids clinical practice regarding client safety with medication management. Clinicians did state that the cost of the kit to their department was not a deterrent to obtaining and using this kit. Overall, clinicians indicated in their responses that the MMS offered the most useful information to inform their practice for clients post-stroke as it assesses "reading, medication knowledge base, problem solving, short-term and prospective memory, and calculations, and lastly performance," all crucial to safety and independence in the realm of medication management ${ }^{13}$.

In current practice, occupational therapists are often not using standardized assessment for assessing medication management capacity in the rehabilitation setting. "Homegrown" assessments lack reliability and validity, and using assessments designed for other professions does not measure occupational therapy outcomes. ${ }^{5,11}$ Occupational therapy practitioners need to adopt new, valid, reliable, easily administered, and client-friendly tools to strive for professional excellence and effectiveness. ${ }^{4}$

This pilot study does give merit and cause for on-going research of this nature. The clinician comments indicate that there is a difference in client performance and utility for each screen. A research study with a greater sample size conducted over a longer period of time could yield data that has more statistical significance. Further research is needed on the general topic of medication management capacity in occupational therapy. Additionally, further research is needed on the use of medication management screens for clients post-stroke.

\section{STUDY LIMITATIONS}

A number of limitations must be considered in the interpretations of this pilot study's findings. First, the sample size for clients was small $(\mathrm{N}=5)$, and even smaller for clinicians $(\mathrm{N}=3)$. Because of the small sample size of clients, some clinicians only conducted the screens on one client; therefore, the depth of information provided in the focus group was limited. Additionally, the focus group was not audio recorded, which could have resulted in some loss of meaning or key phrases provided by clinicians.

Furthermore, the data collected on each screen was only a score; therefore, the content of each test item could not be further analyzed. The S5 screen did not publish norm values to which the overall score could be related, thus leaving the value of the score open to subjective interpretation. Results therefore cannot be generalized, and strong conclusions regarding comparisons between screens cannot be made.

Finally, authors of the S5 have indicated a newer version of the S5 is under study, and literature will be made available soon to the public. Researchers should review the literature and research for this newer version and utilize it in future studies. Future studies should include more client participants to enable clinicians to conduct screening on more than one client for more in depth data.

(C) The Internet Journal of Allied Health Sciences and Practice, 2015 


\section{REFERENCES}

1. AOTA. Occupational Therapy Practice Framework: Domain and Process. American Journal of Occupational Therapy. 2002;56(6):609-39. [PMID 12458855].

2. Creswell JW. Research Design: Qualitative, Quantitative and Mixed Methods Approaches,4e. Thousand Oaks, CA: Sage Publications Inc; 2013.

3. Donovan NJ, Kendall DL, Heaton SC, Kwon S, Velozo CA, Duncan PW. Conceptualizing functional cognition in stroke. Neurorehabil Neural Repair. 2008;22(2):122-35. [PMID 17761809] doi: 10.1177/1545968307306239.

4. Doucet BM. Five years later: achieving professional effectiveness to move neurorehabilitation forward. Am J Occup Ther., 2013;67(5):e106-19. [PMID 23968801] doi: 10.5014/ajot.2013.008417.

5. Doucet BM, Gutman SA. Quantifying function: the rest of the measurement story. Am J Occup Ther. 2013;67(1):7-9. [PMID 23245777]

6. Elliott RA, Marriott JL. Standardised assessment of patients' capacity to manage medications: a systematic review of published instruments. BMC Geriatr. 2009;9:27. [PMID 19594913] doi:10.1186/1471-2318-9-27.

7. Hayes TL, Larimer N, Adami A, Kaye JA. Medication adherence in healthy elders: Small cognitive changes make a big difference. J Aging Health., 2009;21(4):567-80. [PMID 19339680] doi:10.1177/0898264309332836.

8. Kaizer F, Kim A, Van MT, Korner-Bitensky N. Creation and preliminary validation of the screening for self-medication safety post-stroke scale. J Rehabil Med. 2010;42(3):239-45. [PMID 20411219] doi: 10.2340/16501977-0523.

9. Nasreddine Z.Montreal Cognitive Assessment, 2013. Accessed March 11, 2015 at http://www.mocatest.org/.

10. Orwig D, Brandt N, Gruber-Baldini AL. Medication management assessment for older adults in the community. Gerontologist. 2006;46(5):661-8. [PMID 17050757]

11. Radomski MV, Trombly Latham K. Occupational therapy for physical dysfunction, 7e. Philadelphia: Lippincott Williams \& Wilkins; 2014.

12. Robnett RH, Dionne C, Jacques R, Lachance A, Mailhot M. The ManageMed Screening. Clinical Gerontologist. 2007;30(4):1-23. doi:10.1300/J018v30n04_01.

13. Robnett RH, Moyer E. A Quick Assessment of Medication Management Skills (Test Manual). Portland, ME: North East Assessment Tools, LLC; 2007.

14. StrokEngine. 2013a. In depth review of the S5. Accessed March 11, 2015 at http://strokengine.ca/assess/module_s5_indepth-en.html.

15. StrokEngine. 2013b. In depth review of the MoCA. Accessed March 11, 2015 at http://strokengine.ca/assess/module_moca_quick-en.html.

16. Wales K, Clemson L, Lannin NA, Cameron ID. Functional assessments used by occupational therapists with older adults at risk of activity and participation limitations: A systematic review and evaluation of measurement properties. Systematic Reviews. 2012;1(45):2-6. doi:10.1186/2046-4053-1-45.

17. Wolf TJ. Rehabilitation, disability, and participation research: Are occupational therapy researchers addressing cognitive rehabilitation after stroke? American Journal of Occupational Therapy. 2011;65(4):e46-59. doi: 10.5014/ajot.2011.002089. 
Appendix A. Sample of Medication Management Assessments

\begin{tabular}{|l|l|}
\hline \multicolumn{1}{|c|}{ Assessment } & \multicolumn{1}{c|}{ Description } \\
\hline Self-Administration of Medication (SAM) & $\begin{array}{l}\text { Questionnaire, used by nurses to assess a person's competence to } \\
\text { self-administer medications }\end{array}$ \\
\hline Medication Management Ability Assessment (MMAA) & $\begin{array}{l}\text { Used with clients with schizophrenia, assessment in completed } \\
\text { through role-play }\end{array}$ \\
\hline Drug Regimen Unassisted Grading Scale (DRUGS) & $\begin{array}{l}\text { Assesses a highly functioning older adult's ability for self- } \\
\text { medication by looking at ability to identify, access, dose and time } \\
\text { their personal medication routine }\end{array}$ \\
\hline $\begin{array}{l}\text { Medication Management Instrument for Deficiencies in } \\
\text { the Elderly (MedMalDE) }\end{array}$ & $\begin{array}{l}\text { Used for assessment of compliance and management of } \\
\text { medication routine }\end{array}$ \\
\hline Medication Management Tasks (MMT) & $\begin{array}{l}\text { Developed for assessment of adaptive strategies in patients with } \\
\text { dementia }\end{array}$ \\
\hline MedTake Test (MT) & $\begin{array}{l}\text { Assesses understanding of dosage, indications, schedule, and } \\
\text { safety through interview of older adults }\end{array}$ \\
\hline Self-Medication Assessment Tool (SMAT) & $\begin{array}{l}\text { Used by pharmacists to assess deficits in self-management of } \\
\text { medications by looking at function, cognition, self-reported } \\
\text { adherence, medication recall }\end{array}$ \\
\hline
\end{tabular}

(Adapted from Kaizer et al., 2010) 